\title{
Factors and Conditions that Influence the Implementation of Standardized Nursing Care Plans
}

\author{
Inger Jansson ${ }^{*}, 1$, Christel Bahtsevani ${ }^{2}$, Ewa Pilhammar-Andersson ${ }^{3}$ and Anna Forsberg ${ }^{4}$ \\ ${ }^{1}$ School of Social and Health Sciences, Halmstad University, Halmstad, Sweden \\ ${ }^{2}$ Faculty of Health and Society, Malmö University, Sweden \\ ${ }^{3}$ Gothenburg University, The Sahlgrenska Academy, Institute of Health and Care Sciences, Sweden \\ ${ }^{4}$ Gothenburg University, The Sahlgrenska Academy, Institute of Health and Care Sciences, and Department of Surgery, \\ Sahlgrenska University Hospital, Sweden
}

\begin{abstract}
Rationale and Aim: There is a lack of evidence about how to successfully implement standardized nursing care plans (SNCP) in various settings. The aim of this study was to use the "Promotion Action on Research Implementation in Health Services framework" (PARIHS) to explore important factors and conditions at hospital wards that had implemented SNCPs.

Method: We employed a retrospective, cross-sectional design and recruited nurses from four units at a rural hospital and seven units at a university hospital in the western and southern region of Sweden where SNCPs had been implemented. Outcome was measured by means of a questionnaire based on the PARIHS-model.

Result: In total, 137 nurses participated in the study. The main factors that had motivated the nurses to implement SNCPs were that they were easy to understand and follow as well as corresponding to organisational norms. The SNCPs were normally based on clinical experience, although research more frequently formed the basis of the SNCPs at the university hospital. Internal facilitators acted as important educators, who provided reminders to use the SNCP and feedback to the SNCP users. The patient experience was not considered valuable. Those who claimed that the implementation was successful were generally more positive in all measurable aspects. The use of SNCPs was rarely evaluated.

Conclusions: Clinical experience was considered important by the nurses, while they attributed little value to the patient experiences. Successful implementation of research based SNCPs requires internal facilitators with knowledge of evidence-based nursing.
\end{abstract}

Keywords: Implementation, standardized nursing care plans, PARIHS, questionnaire.

\section{INTRODUCTION}

Today, there is a lack of knowledge about how to successfully implement standardized nursing care plans (SNCPs) in various settings in general and hospital wards in particular. There are few previous studies of SNCPs, and those identified focused on effect and utilization as opposed to implementation. Dahm and Wadensten [1] found that nurses perceived that SNCPs increased their ability to provide the same quality of care to all patients and reduced the time spent on documentation as well as unnecessary documentation. Lee and Chang [2] also revealed that SNCPs enhanced the quality of care, as the nurses were reminded of care procedures and time was saved, as they did not need to write everything themselves. However, the SNCP could sometimes be time consuming due to the need to fill in many forms. Another problem was that the SNCP can be inflexible and difficult to apply.

*Address correspondence to this author at the School of Social and Health Sciences, HOS, Halmstad University, Box 823, 30118 Halmstad, Sweden; Tel: 0046-35-167458; Fax: 0046-340-676419; E-mail: Inger.Jansson@hh.se
Opposite to SNCP we know more about Clinical pathways (CPs), which are multi professional plans for the care of a specific group of patients. Scientific knowledge of CPs can be used as a valid foundation for the development of SNCPs. A literature review by Hunter and Segrott [3] suggested that there are several aims behind a CP: minimising healthcare costs by less delays, shortening the length of stay and eliminating task duplication, enhancing the quality of care and implementing evidence-based practice. The above-mentioned authors emphasized that the quality of the knowledge on which CPs are based can vary and that few studies have been performed on how to implement CPs properly. Facilitators seem to be the most common reason for successful implementation although this has not been evaluated. The review also revealed that research about the effect of CPs is limited. A recent multi centre study by Vanhaecht et al. [4] indicated that CPs have a significant impact on the coordination and follow up of the care process. No significant differences were found regarding patient-focused organisation, communication with patient or family or cooperation with primary care.

According to Carpenito [5], an optimal care management system should include CPs, SNCPs and individual care plans 
(ICPs) if necessary. She described an SNCP as a printed care plan that outlines the nursing care to be provided for a patient, family or group [5]. SNCPs originate from an American tradition where there are many written guidelines that focus on care standards [6, 7]. In Sweden, SNCPs have been used since the early 1990s and become a fast growing method for planning patient care. In a Swedish context, the concept of standardized nursing care plans (SNCPs) has been defined by Forsberg and Edlund [8] as:

\section{"A general action plan formulated according to the nursing process. It states the basic level of nursing care and may be used for patients with the same medical diagnosis, for patients undergoing similar medical treatment or patients with similar nursing problems. The plan should include an individualized approach and individualized care plan (ICP) if necessary." p 19.}

In order to provide high quality and safe nursing care on the wards, nurses should be able to "independently apply the nursing process by observation, assessment, nursing diagnosis, planning, interventions and evaluation of patient care" in line with the description of the qualifications required by nurses in Sweden [9]. It is also stated by "The National Board of Health and Welfare" in Sweden that National guidelines should form the basis for the development of institutional practice guidelines and, as a final step, it should be possible to follow the care process for each individual patient by means of documentation [10]. This can be achieved by the nurse formulating an ICP, that meets each patient's individual needs or by choosing one or several SNCPs developed to cover common nursing problems on the ward. According to two Swedish surveys $[11,12]$, the SNCP is used as a clinical guideline, although there is a lack of research behind it. Despite this criticism, the SNCP is a tool that helps nurses to define the mandatory level of nursing care as well as highlighting nursing care plans in patient records, something that has previously been found to be inadequate $[13,14]$.

Implementing SNCP as an everyday and clinically useful tool in a hospital ward might, as with all kinds of implementation, be problematic. Important factors and prerequisites for the use of research results as well as changes in practical working methods in clinical practice can be described on the basis of the Promoting Action on Research Implementation in Health Services (PARIHS) theoretical framework [15]. According to PARIHS, successful change is based on the interaction between evidence, context and facilitator. The PARIHS framework defines evidence as research, clinical experience, patient experience and local data/information (systematically collected and evaluated). The context concerns the environment in which the change is implemented and is divided into culture, leadership and evaluation. Facilitation refers to processes aimed at implementing knowledge in a practical setting and requires a person (the facilitator) to assist the implementation in terms of aims, roles, skills and characteristic features. All of these factors can be placed in a continuum from low to high, and the implementation will be successful if all factors are at the high end. An empirical study conducted to further develop PARIHS revealed that evidence, context and facilitation are key factors and that the content, aims and dynamics of the framework require further discussion [16].

The aim of this study was to use the PARIHS model to explore important factors and conditions at hospital wards that had implemented SNCPs.

\section{Research Questions}

- Which factors and conditions were important when implementing SNCPs at a rural and a university hospital respectively?

- Were there any differences between a rural and a university hospital in terms of the factors and conditions of importance for the implementation of SNCP?

- In what way do these factors and conditions differ between respondents' perception of a successful and an unsuccessful implementation process?

Rural in this study meant a hospital located outside the big cities of Sweden and obliged to provide the local citizens with basic as well as some specialized care.

\section{METHOD}

\section{Sampling and Procedure}

Strategic sampling was performed at a rural and a university hospital in the western and southern region of Sweden in order to achieve variation regarding type of hospital care, level of scientific connection and experience of using SNCPs in daily clinical work. As the aim of the study was to evaluate the implementation of SNCPs, the hospitals were asked which of their departments employed such plans. Based on the response, four care departments were selected from the rural hospital; two medical, one surgical and one infectious disease ward, while seven were selected from the university hospital; two medical, two surgical and two paediatric wards as well as one neuropsychiatric unit. A selection was made of all nurses who worked day/evening and/or night shifts in the various wards $(n=276)$. A list containing the names of the nurses was requested from the respective ward managers. The researchers provided oral information about the study at meetings in the wards, after which the nurses were given an envelope containing written information, a questionnaire and a return envelope, all of which had a code number for the data analysis. When the nurses had filled in the questionnaire, they placed it in the envelope together with a sample of the SNCP referred to in their responses and put the envelope into a sealed box in their ward. The box was later opened by the researcher. A reminder was sent to those who failed to reply within two weeks. The drop-outs were nurses who did not return the questionnaire. Failure to return the questionnaire was interpreted as a refusal to take part in the study.

\section{Data Collection}

A questionnaire was used for data collection, which was originally developed for measuring factors and prerequisites for the implementation of clinical guidelines on the basis of the PARIHS [17]. The questionnaire was revised for the present study in order to make it relevant for SNCPs and thus contained items based on the SNCPs recently used by 
the informants, an example of which they had enclosed in the response envelope. The questions also concerned which methods of implementation were used, whether the implementation were perceived as successful, perceptions of clinical experience, patient experience, culture, leadership, forms of evaluation employed, feedback and the function of facilitators on the ward [17]. A question about evidence was based on the following definition, which was included in the questionnaire:

"Evidence-based here implies that behind the
standardized care plan is a documented
quality norm/knowledge base that describes
the basis of the SNCP. In order for a
foundation to be deemed to be evidence-based,
it must be derived from quality controlled and
systematically searched research results, and
it should be clearly stated whether the
scientific basis is strong, moderate, weak or
insufficient (so-called evidence grading)".

The validity of the original instrument had previously been verified by test subjects who filled in and commented on any lack of clarity in the questionnaire [17]. The revised version used in the present study was not subjected to further validity tests, as we considered that an SNCP is comparable to a form of clinical guideline. The reliability of the instrument was previously investigated by means of a testretest study, in which the questionnaire was filled in by the same people on two separate occasions. The study revealed good concordance for eight questions, moderate concordance for 13 questions and poor concordance for two questions [17]. The revised questionnaire used in the present study had a Cronbach's Alpha of .75 for items involving clinical experience, .79 for items pertaining to patient experiences and .89 for items concerning context and facilitation.

\section{Statistics and Ethics}

The data were mainly at nominal and ordinal level, which generated descriptive statistics or required non-parametric analyses. The Mann-Whitney U-test was employed to analyse differences between two unpaired groups, i.e. the rural hospital versus the university hospital and successful versus unsuccessful implementation. The chi-square distribution was tested in order to compare two sets of nominal data, i.e. sex and successful implementation, while an independent sample test was used to compare age and work experience (years). Spearman's rho correlation test was used to explore the relationship between clinical experiences, patient experiences, context and facilitation. Statistical significance was set at $\mathrm{p}<0.05$.

According to Swedish law at time of the study, ethical approval was not required since the research did not involve patients. However, the ethical aspects of this study are in accordance with the World Medical Association Declaration of Helsinki [18] and the Act concerning the Ethical Review of Research Involving Humans [19]. Permission to carry out the study was granted by the respective ward managers. Participation was voluntary and informed consent was deemed to be obtained by the fact that the informants answered and returned the questionnaire. During the data analysis the names were replaced by a code so that individuals could not be identified. Only the authors of the study had access to the code key.

\section{RESULTS}

\section{Sample and Use of SNCPs in Daily Work}

The total response rate after one reminder was $50 \%$ $(n=137)$. Of the 87 nurses in the rural hospital, $62 \%(n=54)$ returned the questionnaire. In the university hospital, the response rate was $44 \%(n=83)$. In the whole group $(n=137)$ $88 \%(n=120)$ were female and $12 \%(n=17)$ were male. There were more responses from male respondents at the university hospital $(18 \%, \mathrm{n}=15)$ compared with the rural hospital $(2 \%, n=1)(p=.008)$. The mean age was 38 years (range 24-64 years), with no difference between the two hospitals. The mean time since graduation from nursing school was 10 years (0-42 years) with no difference between the hospitals. However, the nurses at the rural hospital had worked longer in their present ward (mean 8.68 years) than their counterparts at the university hospital (mean 5.79 years $)(p=.042)$. Seventy seven percent $(n=105)$ of the respondents worked day or evening shifts, while $13 \%$ $(n=18)$ only worked nights and $10 \%(n=13)$ rotated (one missing answer). Once again no difference between the two hospitals was observed.

Ninety eight per cent of the respondents stated that they used SNCPs in their everyday work. SNCPs were used to the same extent in the hospitals. There was a difference in the number of SNCPs employed by the respondents, as those at the university hospital used 1-3, compared to their counterparts at the rural hospital who made use of an average of 4-6 different SNCPs $(\mathrm{p}=.001)$. The respondents were requested to state the $\mathrm{SNCP}(\mathrm{s})$ they used on the ward, which subsequently formed the basis of their responses to the items in the questionnaire. The staff from the rural hospital referred to 3-8 different SNCPs per ward, while their counterparts from the university hospital only mentioned 12 , which were thus included in the study.

\section{Factors and Conditions Affecting Implementation}

The question about whether the implementation of the named SNCP was successful according to the nurses' own experience was answered by 131 respondents, of whom 73 $\%(n=96)$ stated that it was successful, while $27 \%(n=35)$ considered it unsuccessful (6 missing answers). In this respect there were no differences between the two hospitals.

The basis of the SNCPs that the respondents enclosed with the questionnaire was, according to the respondents perception, mainly clinical experience, $59 \%(n=81)$, and research, $45 \%(n=62)$. Patient experiences were mentioned by $12 \%(n=17)$ of the respondents, while $28 \%(n=39)$ did not know on what the SNCP was based. The informants could choose several alternatives, as their answers could be based on more than one source. A larger number of respondents from the university hospital stated that the SNCP was based on research $(\mathrm{p}=.001)$ and clinical competence $(p=.08)$. However, there was no difference in terms of patient experiences as a basis. Several of the respondents from the rural hospital were unsure about the basis of the SNCP $(p=.03)$. The perceptions of the basis of the SNCPs had no bearing on whether the implementation was considered successful or unsuccessful. 


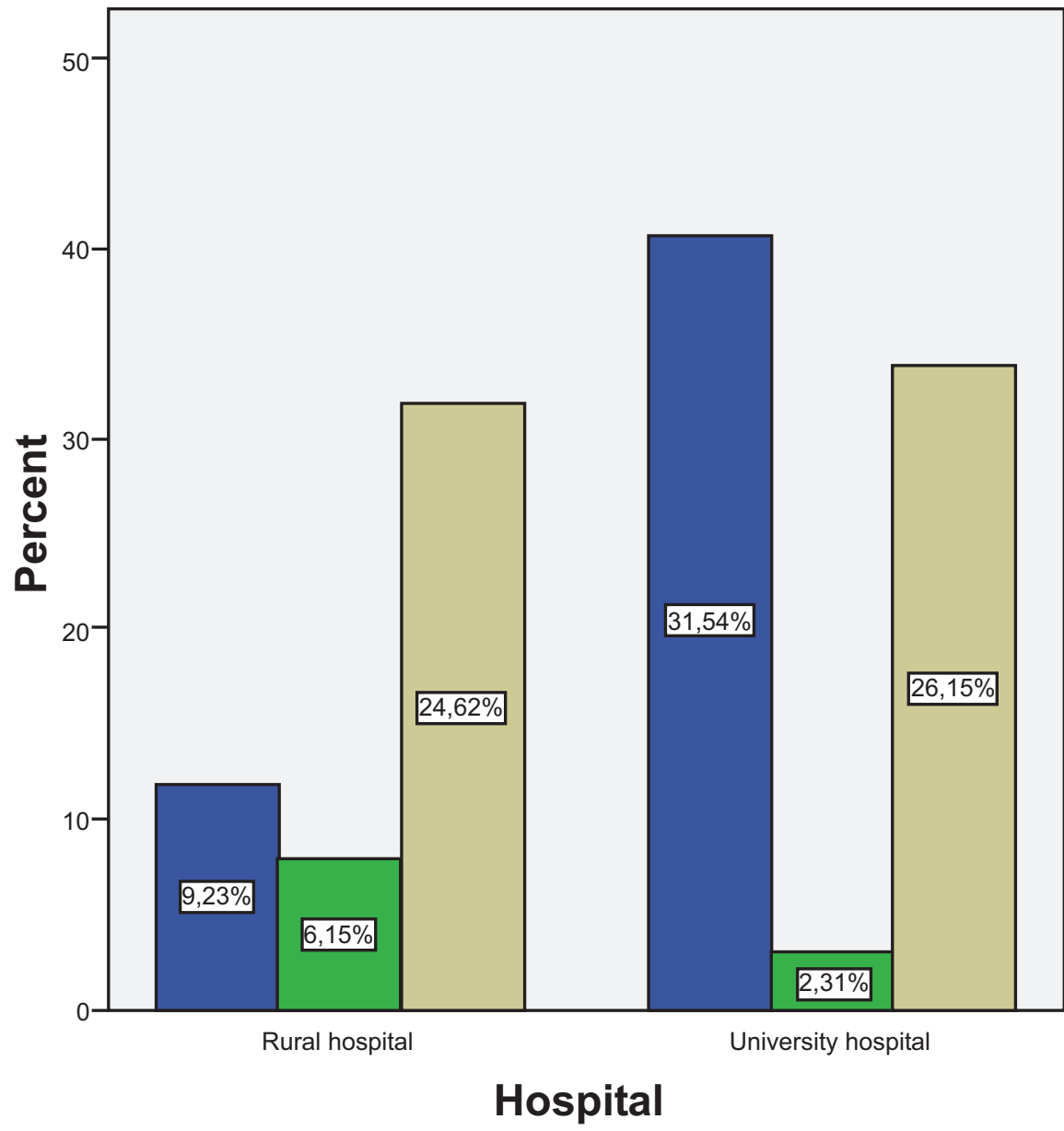

Is the above mentioned SNCp evidence-based?

$\square$ Yes No Don't know

Fig. (1). Percent of informants from the rural hospital and the university hospital who considered that the SNCP was evidence-based in accordance with the definition provided.

Forty one percent $(n=53)$ stated that the SNCP was evidence-based in line with the definition, given in the questionnaire, while $8 \%(n=11)$ reported that it was not. Fifty one percent $(\mathrm{n}=66)$ did not know (seven missing answers). A larger number of the respondents from the university hospital considered that the SNCP was evidencebased $(p=.001)$ (Fig. 1). In this respect there was no difference between those who reported that the implementation was successful and those who stated that it was not.

\section{Factors of Greatest Importance for Implementation of SNCP}

The informants could select several response alternatives to the question about the strengths of the SNCP (Table 1).

A larger number of respondents from the university hospital $(\mathrm{p}=.001)$ stated that it was a strength if the SNCP was research-based. Those who claimed that the implementation of the SNCP was successful were more likely to state that a strength of the SNCP was that it adhered to current standards in the care organisation/current organisational norms/standards $(\mathrm{p}=.012)$ and that it was based on clinical experience $(\mathrm{p}=.006)$.

The three most common implementation strategies were: reminders to apply the new method following the implementation $63 \% \quad(\mathrm{n}=83)$, education before implementation $63 \%(\mathrm{n}=82)$ as well as an internal facilitator $62 \%(\mathrm{n}=82)$ (Table 2$)$. The rural hospital more often used regular feedback as a strategy $(\mathrm{p}=.039)$ compared with the university hospital. Apart from that there was no difference between the two hospitals. A comparison between those who stated that the implementation was successful and those who claimed that it was not revealed that more strategies had been employed by the former, as can be seen in Table $\mathbf{2}$. The informants could indicate several different strategies.

\section{Perceptions of the Evaluation of the SNCP}

Forty eight per cent $(\mathrm{n}=63)$ of the respondents did not know whether the SNCP had been evaluated, while $21 \%$ ( $\mathrm{n}=$ 27) stated that an evaluation had taken place and $30 \%(n=$ $39)$ that it had not (8 missing answers). There was no difference between the two hospitals in this respect. Those who stated that the implementation was successful to a larger extent reported that the SNCP had been evaluated (26\%) $(p=.008)$, compared with those who claimed that it was unsuccessful, where only $6 \%$ said that evaluation had taken place. The evaluation methods are presented in Table 3. It was more common in the rural hospital to evaluate by means of an audit of patient records $(p=.018)$, but other than that there was no difference between the two hospitals. Nor was 
Table 1. The Strengths of the SNCP. Several Alternatives could be Selected. The Differences Between the Total and Individual n Represent Missing Data

\begin{tabular}{|c|c|c|c|}
\hline Total Number of Informants $n=137$ & Yes & No & Don't Know \\
\hline Easy to understand $(\mathrm{n}=133)$ & $93 \%(n=124)$ & $5 \%(n=6)$ & $2 \%(n=3)$ \\
\hline Easy to follow $(n=132)$ & $90 \%(n=119)$ & $8 \%(n=11)$ & $2 \%(n=2)$ \\
\hline In line with organisational norms $(\mathrm{n}=133)$ & $88 \%(\mathrm{n}=116)$ & $5 \%(n=7)$ & $7 \%(n=9)$ \\
\hline Based on clinical experience $(\mathrm{n}=132)$ & $77 \%(\mathrm{n}=101)$ & $2 \%(n=3)$ & $21 \%(\mathrm{n}=28)$ \\
\hline Research based $(n=133)$ & $53 \%(n=70)$ & $7 \%(n=10)$ & $40 \%(n=53)$ \\
\hline Based on patient experience $(n=131$ & $40 \%(n=52)$ & $14 \%(n=19)$ & $46 \%(n=60)$ \\
\hline
\end{tabular}

Table 2. Strategies for the Implementation of an SNCP, and Comparison Between those who Stated that the Implementation was Successful and those who Claimed that it was Not. The Differences Between the Total and Individual n Represent Missing Data. Several Alternatives could be Selected

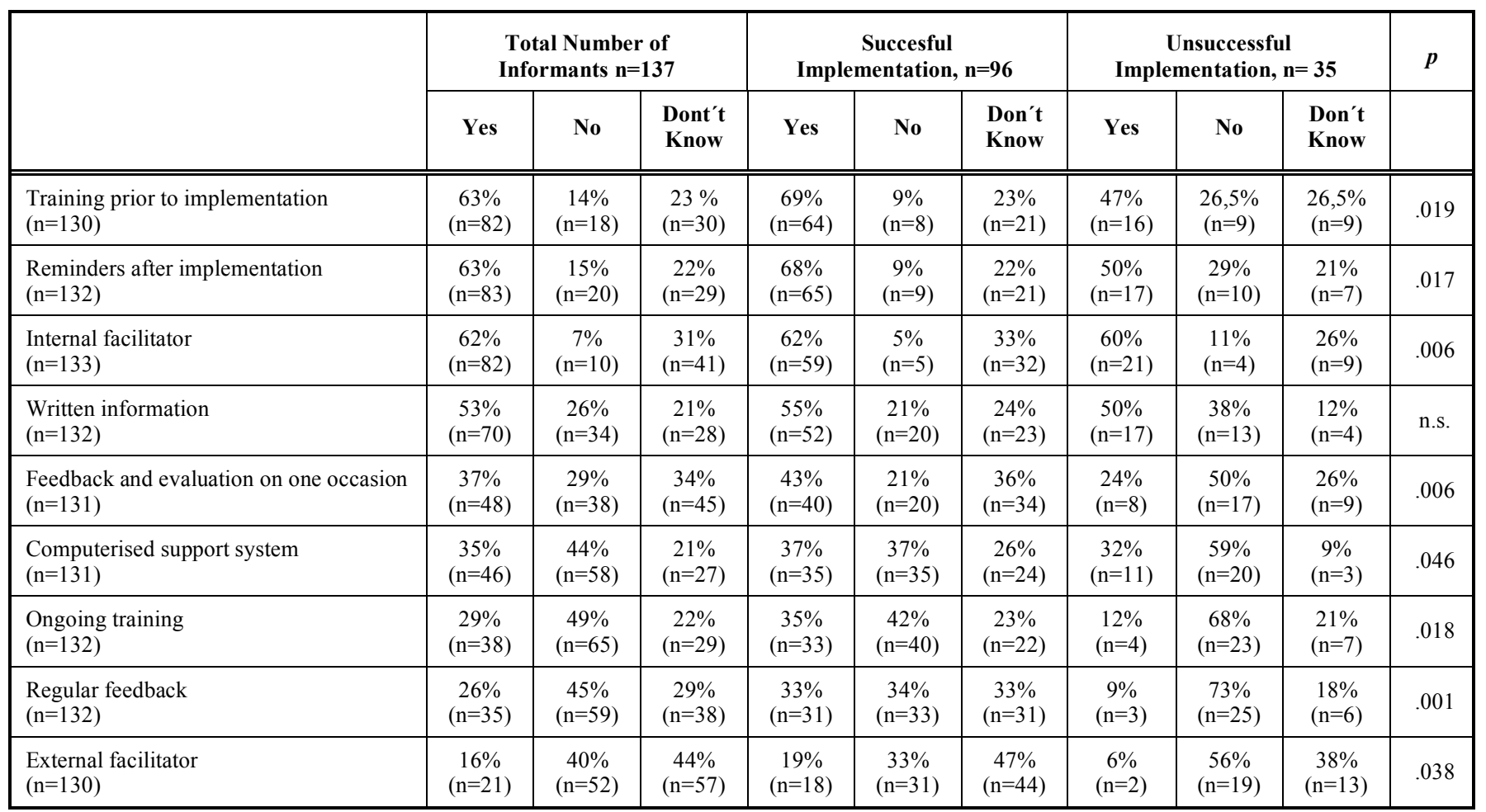

there any difference between respondents who stated that the implementation was successful and those who did not.

\section{The Informants' Perceptions of their Wards}

The questionnaire also included questions about how the respondents perceived their ward in terms of $\mathrm{A}$ : clinical experience, $\mathrm{B}$ : patient experience and $\mathrm{C}$ : context and facilitation. These questions were based on the PARIHS continuum from low to high, where implementation in accordance with PARIHS seems to occur when all three factors are in a high position.

\section{A: Clinical Experience}

More than half of the respondents, $57 \%(n=77)$, stated that they actively discussed/reflected upon the value of clinical experience in their clinical practice. A difference was observed between the hospitals $(p=.028)$ in that $69 \%(n=36)$ of respondents in the rural hospital reported that such discussion and reflection took place compared to $50 \%(\mathrm{n}=$ 41) from the university hospital. Of those who stated that the implementation was successful a larger number, $63 \%$ $(n=59)$, reported that clinical experience was discussed, compared with those who stated that it was not successful, of whom only $40 \%(n=14)$ mentioned discussing clinical experience $(p=.017)$. Table 4 presents the responses to the questions about clinical experience based on the PARIHS continuum from low to high.

\section{B: Patient Experiences}

Among the respondents, $43 \%$ stated that they actively discussed/reflected upon the value of patient experience in their clinical practice. There were no differences concerning hospitals 
or successful/unsuccessful implementation. Table $\mathbf{5}$ presents the responses to the questions about patient experience based on the PARIHS continuum from low to high.

\section{C: Context and Facilitation}

The first six questions in Table 6 concerned the care environment, while the last two addressed facilitating functions based on the PARIHS continuum from low to high.

Table 3. Methods Used for Evaluating the Selected SNCP. The Low Response Rate is Due to the Fact that Only the $21 \%$ of Respondents who Stated that the SNCP had been Evaluated Answered this Question. Several Alternatives could be Selected. The Table Involves All the Informants that Answered the Question on Both Hospitals. The Differences Between the Total and Individual n Represent Missing Data

\begin{tabular}{|c|c|c|c|}
\hline $\begin{array}{c}\text { Total Number of Respondents } \\
\text { who Evaluated the SNCP }(n=27)\end{array}$ & Yes & No & $\begin{array}{c}\text { Don't } \\
\text { Know }\end{array}$ \\
\hline \hline Staff members' experience $(\mathrm{n}=24)$ & $88 \%(\mathrm{n}=21)$ & $4 \%(\mathrm{n}=1)$ & $8 \%(\mathrm{n}=2)$ \\
\hline Patient records $(\mathrm{n}=24)$ & $62 \%(\mathrm{n}=15)$ & $8 \%(\mathrm{n}=2)$ & $29 \%(\mathrm{n}=7)$ \\
\hline Internal registration $(\mathrm{n}=23)$ & $30 \%(\mathrm{n}=7)$ & $26 \%(\mathrm{n}=6)$ & $44 \%(\mathrm{n}=10)$ \\
\hline Patient experiences $(\mathrm{n}=24)$ & $29 \%(\mathrm{n}=7)$ & $33 \%(\mathrm{n}=8)$ & $38 \%(\mathrm{n}=9)$ \\
\hline
\end{tabular}

In summary, the differences between the two hospitals in these three parts containing a total of 18 questions about the respondents' perceptions of their wards revealed that the responses from the staff of the rural hospital scored higher in 15 of these (see Tables 4-6). The responses of the respondents who stated that the implementation was successful had a higher value in 11 out of the 18 questions.
In order to establish which conditions of the PARIHS framework are important when implementing SNCP, we used Spearman's rho to measure the relationship between clinical experience, patient experience and context/facilitation. There was a moderate, positive correlation between clinical experience and context, rho $=.51$ $(\mathrm{p}=.001)$, (32 missing answers) and a weak positive correlation between clinical experience and patient experience, rho $=.39$ (14 missing answers) and between patient experience and context/facilitation: rho $=.44(30$ missing answers).

\section{DISCUSSION}

\section{Study Limitations}

This study involves several limitations that need to be pointed out. First, the response rate was only $50 \%$, which limits the conclusions that can be drawn from the result. The low response rate may be due to the fact that many respondents considered that the questions were difficult and formulated in complicated language, which was mentioned by several of the informants in an open question pertaining to personal views on the questionnaire. The language in the instrument therefore requires simplification, which can be conducted by means of the "think aloud" method [20], where the researcher meets with several persons who are asked to read the questionnaire and "think aloud" when reading and deciding to answer the questions. The fact that the response rate was lower in the university hospital ( $44 \%$ ) than in the rural hospital $(62 \%)$ may be because staff members in the former more often take part in studies, which can lead to questionnaire fatigue.

Secondly, in this study, we asked the respondents about their self reported perceptions of different aspects. Thus, no

Table 4. Respondents' Perceptions of the Present Situation on the Ward in Terms of Clinical Experience. The Scale is Based on the PARIHS Continuum from Low to High, Represented Here by a Scale of 1 to 10. The p-Value Relates to Differences Between the Rural Hospital and the University Hospital. The Results are Given by Median (md) and Percentiles (P25 and P75)

\begin{tabular}{|c|c|c|c|c|c|c|c|c|}
\hline \multicolumn{2}{|c|}{ Questions and Scales (Response Alternatives) } & $\mathbf{N}$ & $\begin{array}{c}\text { md } \\
(\mathbf{P 2 5}, \mathbf{P 7 5})\end{array}$ & n & $\begin{array}{c}\text { md } \\
(\mathbf{P 2 5}, \mathrm{P} 75)\end{array}$ & $\mathbf{n}$ & $\begin{array}{c}\text { md } \\
(\mathbf{P} 25, \text { P75) }\end{array}$ & $\boldsymbol{P}$ \\
\hline \multicolumn{2}{|c|}{$\begin{array}{l}\text { How do you perceive the present situation on the ward in terms of clinical experience? } \\
\text { (scales between } 0.00 \text { and } 10.00 \text { ) }\end{array}$} & & & & & & & \\
\hline $\begin{array}{l}\text { Clinical experience is uncritically and } \\
\text { unsystematically discussed/reflected } \\
\text { upon }\end{array}$ & $\begin{array}{l}\text { Clinical experience is critically and } \\
\text { systematically discussed/reflected upon }\end{array}$ & 130 & $5(5,7)$ & 53 & $6(4,7)$ & 77 & $5(4,7)$ & .003 \\
\hline $\begin{array}{l}\text { There is a lack of judgement of clinical } \\
\text { experience at individual and group level }\end{array}$ & $\begin{array}{l}\text { Clinical experience is judged at individual and } \\
\text { group level }\end{array}$ & 128 & $5(4,7)$ & 53 & $6(5,7)$ & 75 & $5(3,6)$ & .001 \\
\hline $\begin{array}{l}\text { There is a lack of mutual understanding } \\
\text { among different categories of health care } \\
\text { professionals concerning the value of } \\
\text { clinical experience }\end{array}$ & $\begin{array}{l}\text { There is mutual understanding among } \\
\text { different categories of health care } \\
\text { professionals concerning the value of clinical } \\
\text { experience }\end{array}$ & 130 & $7(5,8)$ & 53 & $8(6,8)$ & 77 & $6(5,8)$ & .009 \\
\hline $\begin{array}{l}\text { Clinical experience is valued as the only } \\
\text { form of knowledge in decision making }\end{array}$ & $\begin{array}{l}\text { Clinical experience is valued as one of several } \\
\text { forms of knowledge in decision making }\end{array}$ & 129 & $7(6,8)$ & 53 & $7(6,8)$ & 76 & $7(6,8)$ & .594 \\
\hline
\end{tabular}


Table 5. Respondents' Perceptions of the Present Situation on the Ward in Terms of Patient Experience. The Scale is Based on the PARIHS Continuum from Low to High, Represented here by a Scale of 1 to 10. The p-Value Relates to Differences Between the Rural Hospital and the University Hospital. The Results are Given by Median (md) and Percentiles (P25 and P75).

\begin{tabular}{|c|c|c|c|c|c|c|c|c|}
\hline \multirow{2}{*}{\multicolumn{2}{|c|}{$\begin{array}{c}\text { Questions and Scales (Response } \\
\text { Alternatives) }\end{array}$}} & \multicolumn{2}{|c|}{$\begin{array}{l}\text { Total Number of } \\
\text { Respondents }\end{array}$} & \multicolumn{2}{|c|}{ Rural Hospital } & \multicolumn{2}{|c|}{$\begin{array}{l}\text { University } \\
\text { Hospital }\end{array}$} & \multirow{2}{*}{$p$} \\
\hline & & & $\begin{array}{c}\text { md } \\
(\mathbf{P 2 5}, \mathbf{P 7 5})\end{array}$ & $\mathbf{n}$ & $\begin{array}{c}\text { md } \\
(\mathbf{P 2 5}, \mathbf{P 7 5})\end{array}$ & $\mathbf{n}$ & $\begin{array}{c}\text { md } \\
(\mathbf{P 2 5}, \text { P75) }\end{array}$ & \\
\hline \multicolumn{2}{|c|}{$\begin{array}{l}\text { How do you perceive the present situation on your ward in terms of patient experiences? } \\
\text { (scales between } 0.00 \text { and } 10.00 \text { ) }\end{array}$} & & & & & & & \\
\hline Patient biographies and experience are not used & $\begin{array}{l}\text { Patient biographies and experiences are } \\
\text { used }\end{array}$ & 131 & $7(5,8)$ & 53 & $7(6,8)$ & 78 & $6(4,8)$ & .002 \\
\hline $\begin{array}{l}\text { Patients are not involved in the planning of care } \\
\text { interventions }\end{array}$ & $\begin{array}{c}\text { Patients are involved in the planning of } \\
\text { care actions }\end{array}$ & 131 & $6(4,8)$ & 53 & $6(5,8)$ & 78 & $5(3,7)$ & .009 \\
\hline $\begin{array}{l}\text { Patient experience is valued as the only form of } \\
\text { knowledge in decision making }\end{array}$ & $\begin{array}{l}\text { Patient experience is valued as one of } \\
\text { several forms of knowledge in decision } \\
\text { making }\end{array}$ & 127 & $7(6,8)$ & 52 & $7(6,8)$ & 75 & $7(6,8)$ & .888 \\
\hline
\end{tabular}

objective evidence was provided to demonstrate if the SNCPs were based on research. Nor did we objectively evaluate or analyze the success of the implementation, or the knowledge base behind the SNCPs.

In order to evaluate which parts of the PARIHS were most important for the implementation of SNCPs, the questionnaire would have had to be revised so as to include a separate section for each area, i.e. evidence, context and facilitation, in accordance with the description of the various continuums in the PARIHS [15]. It would also have required more questions to cover all of the parts. Instead, the present questionnaire contained items about the situation on the ward with reference to clinical experience and patient experience, while context and facilitation were combined in one area. The question about how research is discussed on the ward does not appear in this part of the questionnaire.

It would also have been interesting to have conducted a regression analysis aimed at charting the factor(s) that were most closely associated with successful implementation. This could have been achieved if the question about successful/unsuccessful implementation had been answered by means of a rating scale, which could then have been compared with other scales concerning the conditions on the ward. Despite these shortcomings in terms of the inability of the instrument to allow for a comparison between the various PARIHS factors, we believe that the result reveals important aspects associated with the implementation of an SNCP in a hospital ward.

\section{DISCUSSION OF THE RESULTS}

The main results of this study were that the basis of SNCPs mainly involved clinical experience and research, while patient experience was rarely included. Research was more frequently mentioned by the respondents from the university hospital. The strengths of SNCPs were that they were easy to understand and follow as well as being based on the relevant clinical standards and experience, which was reported to a greater extent by those who perceived the implementation as successful. The most common implementation strategies were: reminders to apply the new method after implementation and an internal facilitator. The rural hospital more often used regular feedback as a strategy. Those who reported successful implementation reported that more strategies had been used compared to their counterparts who stated that the implementation was unsuccessful. Only $21 \%$ of the respondents stated that the SNCP had been evaluated, of whom a majority considered the implementation successful. The most common form of evaluation was based on the clinical experience of the staff as well as on patient records, which were employed to a greater degree in the rural hospital. The value of clinical experience was discussed more frequently than the value of patient experience and such discussions were more common in the rural hospital. Those who reported successful implementation were of the opinion that it was discussed to a greater extent.

The main reason for using SNCPs is to enhance the quality of care and implement evidence-based practice. The basis of the SNCPs referred to by the informants in this study mainly comprised clinical experience followed by research, while patient experience was rarely mentioned. According to the PARIHS framework, these three factors can be considered evidence [15]. As a result of a major review, Scott and McSherry [21] defined evidence-based nursing as: 
Table 6. Respondents' Perceptions of the Present Situation on the Ward in Terms of Context and Facilitation. The Scale is Based on the PARIHS Continuum from Low to High, Represented here by a scale of 1 to 10 . The p-Value Relates to Differences Between the Rural Hospital and the University Hospital. The Results are Given by Median (md) and Percentiles (P25 and P75)

\begin{tabular}{|c|c|c|c|c|c|c|c|c|}
\hline \multirow{2}{*}{\multicolumn{2}{|c|}{ Questions and Scales (Response Alternatives) }} & \multicolumn{2}{|c|}{$\begin{array}{l}\text { Total Number } \\
\text { of Respondents }\end{array}$} & \multicolumn{2}{|c|}{ Rural Hospital } & \multicolumn{2}{|c|}{$\begin{array}{l}\text { University } \\
\text { Hospital }\end{array}$} & \multirow{2}{*}{$\boldsymbol{P}$} \\
\hline & & $\mathbf{n}$ & $\begin{array}{c}\text { md } \\
(\mathbf{P 2 5}, \mathbf{P 7 5})\end{array}$ & n & $\begin{array}{c}\text { md } \\
(\mathbf{P 2 5}, \mathbf{P 7 5})\end{array}$ & & $\begin{array}{l}\text { md } \\
(\mathbf{P 2 5}, \text { P75) }\end{array}$ & \\
\hline \multicolumn{9}{|c|}{$\begin{array}{l}\text { How do you perceive the present situation on your ward in terms of the context of care, } \\
\text { forms of evaluation and the function of the facilitator? (scales between } 0.00 \text { and } 10.00 \text { ) }\end{array}$} \\
\hline 0.00 & 10.00 & & & & & & & \\
\hline The context is not receptive to change & The context is receptive to change & 136 & $7(6,8)$ & 54 & $8(6,9)$ & 82 & $7(5,8)$ & .001 \\
\hline $\begin{array}{l}\text { The context is characterized by a culture that } \\
\text { promotes a task driven organisation }\end{array}$ & $\begin{array}{c}\text { The context is characterized by a } \\
\text { culture that promotes a learning } \\
\text { organization }\end{array}$ & 134 & $6(5,8)$ & 53 & $7(5,9)$ & 81 & $6(4,7)$ & .006 \\
\hline $\begin{array}{l}\text { The context is characterized by traditional } \\
\text { (command and control) leadership }\end{array}$ & $\begin{array}{l}\text { The context is characterized by } \\
\text { transformational leadership }\end{array}$ & 134 & $6(3.75,7)$ & 54 & $7(5,8)$ & 80 & $5(3,7)$ & .001 \\
\hline $\begin{array}{l}\text { Clinical, performance, economic and experience } \\
\text { evaluations are dependent on single } \\
\text { as opposed to multiple methods }\end{array}$ & $\begin{array}{l}\text { Multiple methods are used for } \\
\text { clinical, performance, economic and } \\
\text { experience evaluations }\end{array}$ & 122 & $6(4,7)$ & 48 & $6.5(5,8)$ & 74 & $5(3,6)$ & .001 \\
\hline $\begin{array}{l}\text { There is a lack of feedback concerning individual, } \\
\text { team and system performance }\end{array}$ & $\begin{array}{l}\text { There is feedback on individual, team } \\
\text { and system performance }\end{array}$ & 133 & $6(4,7)$ & 53 & $7(5,8)$ & 80 & $5(3,6)$ & .001 \\
\hline $\begin{array}{l}\text { There is a lack of facilitators, or facilitation methods } \\
\text { are inappropriate }\end{array}$ & $\begin{array}{l}\text { Availability of facilitators and } \\
\text { appropriate facilitation methods }\end{array}$ & 129 & $6(4,7)$ & 51 & $7(5,7)$ & 78 & $5(4,7)$ & .01 \\
\hline $\begin{array}{l}\text { The function and role of the facilitator aims at doing } \\
\text { for others (for example searching for research } \\
\text { literature) }\end{array}$ & $\begin{array}{c}\text { The function and role of the facilitator } \\
\text { aims at enabling others (for example } \\
\text { teaching them how to search for } \\
\text { literature) }\end{array}$ & 120 & $5(3,7)$ & 47 & $5(4,7)$ & 73 & $5(3,7)$ & .824 \\
\hline
\end{tabular}

"An ongoing process by which evidence, nursing theory and the practitioners' clinical expertise are critically evaluated and considered, in conjunction with patient involvement, to provide delivery of optimum nursing care for the individual" [21, 2008 p. 1089].

In order to obtain the best possible nursing evidence, the supporting factors have to be used in a particular internal sequence according to Scott and McSherry [21], who developed a model for evidence-based nursing, in which the first level includes national guidelines, policies and empirical research. The second level comprises local policies, clinical experience and nursing theories. The third level involves the use of practitioners' knowledge and experience, which are critically evaluated with reference to levels one and two, before continuing with level four, where the patient is involved in the decision-making and evaluation of care. Less then half of the respondents in this study stated that research formed the basis for the SNCP, which corresponds to the first level in the above-mentioned model. This was more often reported by the respondents from the university hospital, which may possibly be explained by the fact that research is more frequently discussed there, since an inherent demand in the hospital is the production of new knowledge, or that those who developed the SNCPs were more familiar with how to search for research results. Even in surveys [11, $12]$ in which a large number of SNCPs were examined, the lack of a research knowledge base was obvious.

It must be regarded as serious that nurses in the units studied did not consider research to be a priority. This agrees with Forsman et al. [22], who revealed that, one year after graduation, nurses were low users of research and even more so three years after graduation. Likewise, Rycroft-Malone et al. [16] found that the nurses did not consider research important. The nurses in a Swedish study [23] stated that nurses should not talk too much about the use of research in clinical practice if they wish to maintain their status within the group, as the attitude to research is rather reserved. Since the nursing education today is highly academic and based on research, these results are worrying and require reflection. The present study also revealed that $61 \%$ of the respondents from the rural hospital did not know whether the SNCP was evidence based compared to $43 \%$ from the university hospital. Dahm \& Wadensten's [1] study also demonstrated that over half of the nurses were not aware that their SNCPs were evidence-based, described as"best available knowledge". 
Many of the respondents believed that level two of the above-mentioned model was extremely valuable, as clinical experience usually constituted the basis of the SNCP, which was considered a strength. The respondents also reported that a strength of the SNCP was that it adhered to the norms of the ward and was easy to follow, which is compatible with this level where local policies are also found.

The last level, where patient involvement and evaluation are in focus, was revealed to have very little impact on the implementation and evaluation of SNCPs. Our study demonstrates that few respondents believed that patient experience was used in the development of SNCPs, as does a study of clinical pathways [3]. There is a risk that failure to consider patient experience at the planning and development stage may have been due to a negative perspective on patient knowledge, which resulted in patients not being included in the use of the SNCP.

We believe that this is worrying, as one of the main benefits of SNCPs (and CPs) is that they enable the nurse to discuss the treatment goals and interventions with their patients, thereby ensuring their involvement in the care. Patient participation is also a basic prerequisite of nursing, as the patients' narratives and subjective experiences should form the basis of the nursing care. Nor were the patients involved in the evaluation of the SNCPs. In general, evaluation was rare and, when carried out, staff members' experience and patient records were usually employed.

Scott and McSherry [21] described their model as a method for implementing evidence-based nursing in practice. However, our study reveals that nurses find it difficult to assimilate the first level, which comprises research, among other things in the form of national guidelines. This requires facilitators who highlight and elucidate evidence as a basis for the nurses' continuing work of developing the SNCP in their own context.

The most common implementation strategy was the use of internal facilitators. A similar number reported that training before as well as reminders after the implementation were used, which leads us to the conclusion that this had been organised by the internal facilitator. External facilitators were only used to a small extent, thus we can conclude that external training rarely took place. Instead, the training was conducted within the context of the workplace, which indicates that this is the best method for implementing SNCPs. Those who stated that the implementation was successful also reported the use of more strategies than their counterparts who claimed that the implementation was unsuccessful, which agrees with the results of a review by Grimshaw et al. [24], who revealed that multifaceted interventions were more effective than single ones.

The questions about how the respondents perceived their wards in terms of clinical and patient experience in addition to context and facilitation revealed that despite a significant correlation between the three parts measured, the result was moderate, which would have been interesting to explore in greater detail. Based on the PARIHS continuum, the respondents from the rural hospital scored higher on 15 out of the 18 questions pertaining to the general conditions on the ward (see Tables 4-6), but we are unable to explain why this is so. Those who stated that the implementation was successful scored higher on 11 of the 18 questions based on the PARIHS continuum (Tables 4-6). The fact that those who considered that the implementation was successful responded in a more positive way to the questions where a difference in responses was measurable is difficult to evaluate but may be due to a more critical attitude and higher expectations on the part of their counterparts who stated that the implementation was unsuccessful. A follow-up question as to why they considered the implementation unsuccessful would have been useful.

\section{IMPLICATIONS AND FURTHER RESEARCH}

Our study demonstrates that in order for SNCPs to be implemented in clinical practice, they need to be easy for the nurses to assimilate, thus enabling their use as a tool in clinical practice. It is also important for internal facilitators to be supported by the nurse leaders in order to ensure time and resources for the implementation work. It takes time and knowledge to develop an SNCP, but it increases the quality of the operations. Therefore such work should be prioritised and co-ordinated to avoid every hospital or unit carrying out duplicate work. Resources should be devoted to knowledgeable care developers and internal facilitators working jointly to develop SNCPs that can be used within different types of operations. More research is required to explore the reason why patient experience is not considered and how this situation can be improved. We also require more knowledge about why research appears to be of no interest to practising clinical nurses, as well as how their attitude can be changed.

\section{REFERENCES}

[1] Dahm MF, Wadensten B. Nurses' experiences of and opinions about using standardized care plans in electronic health records $-\mathrm{a}$ questionnaire study. J Clin Nurs 2008; 17(16): 2137-45.

[2] Lee T-T, Chang P-C. Standardized care plans: experiences of nurses in Taiwan. J Clin Nurs 2004; 13(1): 33-40.

[3] Hunter B, Segrott J. Re-mapping client journeys and professional identities: A review of the literature on clinical pathways. Int $J$ Nurs Stud 2008; 45(4): 608-25.

[4] Vanhaecht K, De Witte K, Panelle M, Sermeus W. Do pathways lead to better organized care processes? J Eval Clin Pract 2009; 15(5): 782-8.

[5] Carpenito LJ. Nursing diagnosis - application to clinical practice. 8 th ed. Philadelphia Lippincott 2000.

[6] Tucker SM, Canobbio MM, Vargo-Paquette E, Wells MF. Patient Care Standards. Collaborative Planning \& Nursing Interventions. $7^{\text {th }}$ ed. St. Louis: Mosby Inc 2000.

[7] Nettina SM. Ed. Manual of Nursing Practice. $8^{\text {th }}$ ed. Philadelphia: Lippincott 2006.

[8] Forsberg A, Edlund K. Standardvårdplaner [Standardized nursing care plan]. Lund: Studentlitteratur 2003 [In Swedish].

[9] The National Board of Health and Welfare. Kompetensbeskrivning för sjuksköterskor [Description of the qualifications required by nurses] Stockholm: Socialstyrelsen. Liber, Allmänna förlaget 2005 [In Swedish].

[10] The National Board of Health and Welfare. God vård - om ledningssystem för kvalitet och patientsäkerhet i hälso- och sjukvården [Good care - about management systems for quality and patient safety in health care]. 2006 [In Swedish].

[11] The National Board of Health and Welfare. Standadvårdplaner (SVP) - en kartläggning av förekomsten av SVP I akutsjukvården [Standardized Care Plans (SNCP) - a survey of SNCPs in acute care settings]. Article no 2006-124-4. Available from: http://www.socialstyrelsen.se/Lists/Artikelkatalog/Attachments/967 7/2006-124-4V்revV்20061244.pdf [Cited 12 jan 2010] [In Swedish]. 
[12] Olsson PT, Peterson H, Willman A, Gardulf A. Standardized care plans in Swedish Health care: Their quality and the extent to which they are used. Scand J Caring Sci 2009; 18: 23(4): 820-5.

[13] Ehrenberg A, Birgersson C. Nursing documentation of leg ulcers: adherence to clinical guidelines in a Swedish primary health care district. Scand J Caring Sci 2003; 17(3): 278-84.

[14] Muller-Staub M, Lavin MA, Needman I, Achterberg T. Nursing diagnosis, interventions and outcomes - application and impact on nursing practice: systematic review. J Adv Nurs 2006; 56(5): 51431.

[15] Rycroft-Malone J. The PARIHS Framework - A framework for guiding the implementation of evidence based practice. J Nurs Care Qual 2004; 19(4): 297-304.

[16] Rycroft-Malone J, Harvey G, Seers K, Kitson A, McCormack B, Titchen A. An exploration of the factors that influence the implementation of evidence into practice. J Clin Nurs 2004; 13(8): 913-24.

[17] Bahtsevani C, Khalaf A, Willman A, Östman M. Developing an instrument for evaluating implementation of clinical practice guidelines: a test-retest study. J Eval Clin Pract 2008; 14(5): 83946.
[18] World Medical Association Declaration of Helsinki. In: The Swedish Research Council's guidelines for the ethical evaluation of medical research on humans. Stockholm: Medicinska forskningsrådet (MFR) 2003. pp. 99-104 [In Swedish].

[19] SFS 2003:460. Lag om etikprövning av forskning som avser människor [The Act concerning the Ethical Review of Research Involving Humans]. 2003 (In Swedish).

[20] Fonteyn ME, Kuipers B, Grobe SJ. A description of the think aloud method and protocol analysis. Qual Health Res1993; 3(4): 430-41.

[21] Scott K, McSherry R. Evidence-based nursing: clarifying the concepts for nurses in practice. J Clin Nurs 2008; 18(8): 1085-95.

[22] Forsman H, Gustavsson P, Ehrenberg A, Rudman A, Wallin L. Research use in clinical practice - extent and patterns among nurses one and three years postgraduation. J Adv Nurs 2008; 65(6): 1196-206.

[23] Holmberg J, Eriksson H. In Principle but never again -Nurses' reasoning about nursing research and research utilisation. Vård I Norden 2009; 3(29): 4-7 [In Swedish].

[24] Grimshaw J, Eccles M, Tetroe J. Implementing clinical guidelines; Current evidence and future implications. J Contin Educ Health Prof 2004; 24(Suppl 1): 31-7.

(C) Jansson et al.; Licensee Bentham Open.

This is an open access article licensed under the terms of the Creative Commons Attribution Non-Commercial License (http://creativecommons.org/licenses/by$\mathrm{nc} / 3.0 /$ ) which permits unrestricted, non-commercial use, distribution and reproduction in any medium, provided the work is properly cited. 